\title{
Epidemiology and Predictors of Hospital Outcomes of Critically Ill Pediatric Oncology Patients: A Retrospective Study
}

\author{
Shilpushp J Bhosale ${ }^{1} \odot$, Malini Joshi² ${ }^{\oplus}$, Vijaya P Patil ${ }^{3} \odot$ Amol T Kothekar $^{4} \odot$, Sheila Nainan Myatra ${ }^{5} \odot$, Jigeeshu V Divatia ${ }^{\circ}$,
} Atul P Kulkarni ${ }^{7}$

\begin{abstract}
Background: The number of pediatric oncology patients admitted to the intensive care unit (ICU) has increased, and their hospital outcomes are improving. Since scarce data are available about this patient population, we conducted this retrospective study to evaluate the epidemiology and predictors of hospital outcomes.

Materials and methods: We included all children with cancers who were admitted to our ICU over 1 year. We excluded children admitted after elective surgery and those following bone marrow transplant. We collected data about demographics, admission diagnosis, type of malignancies, and ICU interventions. The primary outcome was the hospital outcome. The secondary outcomes were ICU length of stay (LOS), and ICU and hospital mortality. We analyzed the predictors of hospital outcome.

Results: Two hundred pediatric oncology patients were admitted from November 1, 2014 to October 30, 2015. Seventy-eight children had solid organ malignancies, and the rest had hematological malignancies. Hematooncology malignancy patients had significantly higher hospital mortality than those with solid organ malignancies. $(61.5 \mathrm{vs} 34.6 \%, p=0.015)$. On multivariate regression analysis, mechanical ventilation [odds ratio (OR), $14.64 ; 95 \%$ confidence interval $(\mathrm{Cl}): 1.23-165.05 ; p<0.030]$, inotropes (OR, $9.81 ; 95 \% \mathrm{Cl}: 1.222-78.66 ; p<0.032)$, and the presence of coagulopathy (OR, 3.86; $95 \% \mathrm{Cl}$ : 1.568-9.514; $p<0.003)$ were independent predictors of hospital mortality.

Conclusion: In this retrospective cohort of 200 children with malignancies, we found that children with hematologic cancer had significantly higher hospital mortality as compared to those with solid tumors. The need for mechanical ventilation, use of inotrope infusion, and coagulopathy were independent predictors of mortality.

Keywords: Critical care oncology, Hematooncology, Hospital mortality, ICU mortality, ICU outcomes, Intensive care in pediatric cancer, Pediatric cancers, Solid tumors.

Indian Journal of Critical Care Medicine (2021): 10.5005/jp-journals-10071-23984
\end{abstract}

\section{INTRODUCTION}

Therapeutic advances in pediatric cancer have significantly improved the overall survival; however, this comes at the cost of increasing intensive care unit (ICU) admissions. ${ }^{1-5}$ Nearly $40 \%$ of pediatric oncology patients require ICU admission during the course of their treatment. ${ }^{6}$ The mortality rate in children without cancer admitted to the pediatric intensive care unit (PICU) which was $11 \%$ in 1982 decreased to around $5 \%$ in 2006 and by 2015 had further decreased to a mere $2.7 \%{ }^{7}$ This is in glaring contrast to a nearly $20 \%$ mortality in pediatric patients with cancer in 2019, which has more or less remained constant, when postoperative patients were excluded. ${ }^{6}$ Over the years, the commonest reasons for ICU admissions in pediatric cancer patients have been sepsis or septic shock and respiratory failure, with mortality of 20 to $84 \% .{ }^{8-10}$ Various risk factors for mortality in children with cancer have been identified, such as sepsis, need for mechanical ventilation, renal replacement therapy, and history of bone marrow transplant. ${ }^{10-12}$ Pediatric patients with hematological malignancies have greater admission illness severity, infection rates, and mortality as compared to those with solid tumors. ${ }^{10}$ We conducted this analysis to assess the clinical characteristics and predictors of hospital outcomes of pediatric cancer patients admitted to the ICU.

\section{Materials and Methods}

We retrospectively analyzed the data of all unplanned pediatric oncology patients admitted to our ICU, at a tertiary referral cancer
1,4,5,7 Division of Critical Care Medicine, Department of Anaesthesia, Critical Care and Pain, Tata Memorial Hospital, Homi Bhabha National Institute, Mumbai, Maharashtra, India

2,3,6 Department of Anaesthesia, Critical Care and Pain, Tata Memorial Center, Homi Bhabha National Institute, Mumbai, Maharashtra, India Corresponding Author: Atul P Kulkarni, Division of Critical Care Medicine, Department of Anaesthesia, Critical Care and Pain, Tata Memorial Hospital, Homi Bhabha National Institute, Mumbai, Maharashtra, India, Phone: +91 9869077526, e-mail: kaivalyaak@ yahoo.co.in

How to cite this article: Bhosale SJ, Joshi M, Patil VP, Kothekar AT, Myatra SN, Divatia JV, et al. Epidemiology and Predictors of Hospital Outcomes of Critically III Pediatric Oncology Patients: A Retrospective Study. Indian J Crit Care Med 2021;25(10):1183-1188.

Source of support: Nil

Conflict of interest: None

center over an 1-year period (November 01, 2014 to October 30, 2015). The study was approved by the Institutional Ethics Committee, which waived off the need for informed consent.

We included data of all patients who were $<18$-year-old. The patients were broadly categorized into a solid organ and hematological malignancies, using the International Classification of Childhood Cancer-3. We excluded planned postoperative

() The Author(s). 2021 Open Access This article is distributed under the terms of the Creative Commons Attribution 4.0 International License (https://creativecommons. org/licenses/by-nc/4.0/), which permits unrestricted use, distribution, and non-commercial reproduction in any medium, provided you give appropriate credit to the original author(s) and the source, provide a link to the Creative Commons license, and indicate if changes were made. The Creative Commons Public Domain Dedication waiver (http://creativecommons.org/publicdomain/zero/1.0/) applies to the data made available in this article, unless otherwise stated. 
admissions and those who had undergone bone marrow transplant.

Our ICU is a mixed adult and pediatric ICU. Acutely ill children from oncology are admitted to the ICU if they require respiratory, or any other organ support or intense monitoring. We collected the demographic data, ICU admission diagnosis (respiratory failure, cardiac failure, etc., see Appendix I for definitions), type of malignancies, ICU interventions required such as mechanical ventilation, vasopressor, and inotropic support, dialysis, and need for transfusion of blood and blood products. The primary endpoint was the hospital outcome. The secondary outcomes were ICU and hospital length of stay (LOS), and ICU mortality. We also analyzed the predictors of hospital outcome.

\section{Statistics}

Categorical variables are presented as counts (percentage) and compared using Fisher's exact test or chi-square test as applicable. For continuous variables, Student's $t$ test was used. Univariate analysis was performed to identify factors predicting ICU mortality, and variables with a $p$ value $<0.2$ were used for multivariable analysis. Multivariable logistic regression analysis was performed by backward stepwise selection of variables. SPSS software version 21 (IBM, Chicago) was used for statistical analysis. $p$ value of $<0.05$ was considered statistically significant.

\section{Results}

We collected data of 200 pediatric oncology patients. Of these, 78 patients had solid organ malignancies ( 83 admissions) and the remaining 122 had hematological malignancies (142 admissions). There were 136 males, and the median age of the patients was 8 (0.5-18) years. The median ICU LOS was 4 (1-31) days, while the median hospital LOS was 12 (1-76) days. Table 1 shows the ICU admission diagnosis for this cohort.

The ICU and hospital mortality in our study cohort were 45 and $51 \%$, respectively. The hospital mortality of patients with hematological malignancies was significantly higher than those with solid organ malignancies ( 61.5 vs $34.6 \% ; p=0.015$ ). The mean duration of ICU stay was higher for survivors as compared to nonsurvivors (5.30 \pm 5.46 vs $3.74 \pm 3.24$ days; $p=0.07)$. The mean duration of hospital stay was also higher for survivors as compared to non-survivors [19.65 ( \pm 14.53 ) days vs 10.91 days $( \pm 9.32) ; p<0.01$ ] The hematological malignancy patients had significantly higher ICU mortality than those with solid organ malignancies (54.1 vs $30.8 \% ; p=0.026$ ). On multivariate regression analysis, the need for ventilation, inotropes, and the presence of coagulopathy were independent predictors of hospital mortality (Table 2).

Forty-three percent of patients had presented to ICU with respiratory failure, and $78 \%$ of these patients required mechanical ventilation. ICU mortality in ventilated patients was significantly higher than patients who did not need ventilation (55.8 vs $9.1 \%$; $p<0.001)$. The mortality rates for ventilated patients were $54 \%$ for hematologic and $30.8 \%$ for solid tumors patients. The duration of ventilation for survivors was 3.7 ( \pm 3.24 ) (range 1-14) days $(p=0.031)$. Thirty-five patients were initially offered noninvasive ventilation (NIV), of which 19 patients subsequently required invasive mechanical ventilation (IMV). The mean duration of NIV was 1.8 ( \pm 1.55 ) days (Table 3 ). The incidence of septic shock at admission was higher in patients with hematological malignancy, and the mortality in these patients was $50 \%$. Seventy-eight patients (39\%) had coagulopathy at admission. The mean international normalized ratio (INR) of 77 patients (data missing for one patient) was 2.0549 ( \pm 1.79 ); of these, 26 patients needed frequent fresh frozen plasma (FFP) transfusions and 8 (32.0\%) patients died. The mean platelet count was $47.70 \times 10^{3}( \pm 53.77) / \mathrm{mm}^{3}$ (minimum, $3 \times 10^{3} / \mathrm{mm}^{3}$; max, $319 \times 10^{3} / \mathrm{mm}^{3}$ ); 62 patients were transfused random donor platelets or single donor platelets as per availability, and 40 (64.5\%) patients died. Twenty-one children with solid tumors had neutropenia, of which 10 (47.6\%) died in the hospital; while 43 children with hematological malignancies had neutropenia, and $24(55.8 \%)$ died in the hospital $(p<0.44)$.

\section{Discussion}

In this retrospective analysis of the largest cohort of pediatric oncology patients from a single center in India, we found that children with hematologic cancer had significantly higher hospital mortality as compared to those with solid tumors. Children who required mechanical ventilation, inotrope infusion, and had coagulopathy had higher mortality, and we found these factors to be independent predictors of mortality.

In this analysis, we found overall ICU and hospital mortality of 45 and $51 \%$, respectively. These figures are much higher than those in

Table 2: Independent predictors of ICU mortality on multivariate analysis

\begin{tabular}{lccc}
\hline & \multicolumn{3}{c}{$95 \%$ confidence } \\
& Odds ratio & interval & p value \\
\hline Mechanical ventilation & 14.64 & $1.23-165.05$ & 0.030 \\
Inotropes & 9.81 & $1.222-78.66$ & 0.032 \\
Coagulopathy & 3.86 & $1.568-9.514$ & 0.003 \\
\hline
\end{tabular}

Table 1: ICU admission diagnosis

\begin{tabular}{lccc}
\hline ICU admission diagnosis & $\begin{array}{c}\text { Total } \\
\text { complications, } n\end{array}$ & $\begin{array}{c}\text { Hematological } \\
\text { malignancies, } n(\%)\end{array}$ & $\begin{array}{c}\text { Solid organ } \\
\text { malignancies, } n(\%)\end{array}$ \\
\hline Acute respiratory failure & 86 & $61(70.9)$ & $25(29.1)$ \\
Encephalopathy & 46 & $29(63.1)$ & $17(36.9)$ \\
Cardiac failure & 19 & $8(42.1)$ & $11(57.9)$ \\
Postop. patients & 18 & $1(5.6)$ & $17(94.4)$ \\
Septic shock & 18 & $14(77.8)$ & $4(22.2)$ \\
Coagulopathy & 78 & $56(71.79)$ & $22(28.21)$ \\
Cardiac arrest & 4 & $4(100)$ & 0 \\
Renal failure & 3 & $1(33.3)$ & $2(66.7)$ \\
Other & 6 & $4(66.7)$ & $2(33.3)$ \\
\hline
\end{tabular}

\#Unplanned admission from wards for postoperative patients for ventilator support and/or vasopressors;

^Admissions after cardiac arrests in wards (code blue patients); for definitions, see Appendix I 
Hospital Outcomes of Critically Ill Pediatric Oncology Patients

Table 3: Interventions in the ICU and mortality ( $N=200$ patients)

\begin{tabular}{lcccc}
\hline Interventions & Total, $n$ & Survived, $n(\%)$ & Mortality, $n(\%)$ & $p$ value \\
\hline Ventilation & 156 & $70(44.8)$ & $86(55.8)$ & $<0.001$ \\
NIV & 35 & $21(60)$ & $14(40)$ & 0.472 \\
Invasive ventilation & 121 & $49(40.4)$ & $72(59.6)$ & $<0.001$ \\
Vasopressors & 76 & $20(25.3)$ & $56(74.7)$ & $<0.001$ \\
Inotropes & 15 & $2(13.3)$ & $13(86.7)$ & $<0.001$ \\
Renal replacement therapy & 14 & $3(21.4)$ & $11(78.6)$ & 0.012 \\
PRBC transfusions & 53 & $25(48.2)$ & $28(52.8)$ & 0.181 \\
FFP transfusions & 38 & $24(63.15)$ & $14(36.84)$ & 0.261 \\
Platelet transfusions & 97 & $61(62.88)$ & $36(37.11)$ & 0.029 \\
\hline PRBC
\end{tabular}

PRBC, packed red blood cells; FFP, fresh frozen plasma

non-oncology admissions to PICUs. An early study from Spain found that the overall mortality in non-cancer general pediatric patients was 5.4 ( \pm 3.2$) \%{ }^{12}$ In 2015, Butt et al., in an editorial, noted that the mortality of pediatric patients in PICUs has significantly decreased over time from 11 (1982) to $2.7 \%$ (2015). ${ }^{7}$ However, pediatric patients with malignancy are immunodeficient either due to disease or due to treatment and are susceptible to repeated infections. A recent Brazilian study found that children with hematological malignancies received corticosteroids more often ( 68.4 vs $50.3 \% ; p=0.02)$ and had higher proportionate use of chemotherapy (84.2 vs $67.3 \%$; $p=0.01$ ) as compared to those with solid tumors. As a consequence, neutropenia ( 54.4 vs $21.6 \% ; p<0.001$ ) and mucositis ( 29.8 vs $12.4 \%$; $p<0.001$ ) occurred more frequently in these children, making them more susceptible to bloodstream infections. ${ }^{13}$

In our patient cohort, children with hematological malignancies had significantly higher ICU (54 vs 30.8\%, respectively; $p=0.026$ ) and hospital mortality (61.5 vs $34.9 \%$, respectively; $p=0.015$ ) as compared to those with solid organ malignancies. This is consistent with several other reports. Zinter et al. reported that children with hematological malignancies had twice the mortality as compared to those with solid tumors (9.6 vs 4.5\%), relative risk $(\mathrm{RR})=2.1(95 \% \mathrm{Cl}: 1.8-2.5 ; p<0.001) .{ }^{11}$ The patients with hematological malignancies were sicker (PRISM 3 score, 8 vs $2 ; p<0.001$ ) and had higher incidence of sepsis ( 27 vs $9 \%$, $\mathrm{RR}=2.9 ; 95 \% \mathrm{Cl}: 2.6-3.1 ; p<0.001)$. Apart from this, diagnosis of acute myeloid leukemia and history of bone marrow transplant were also independently associated with mortality. An Egyptian study reported similarly higher mortality rates in children with hematological malignancies ( 46 vs $22 \% ; p=0.002$ ). The authors attributed this difference to a higher level of immunosuppression in children with hematological malignancies and those with solid tumors being admitted for postoperative care or intense monitoring..$^{10}$ Dalton et al. in a multicenter retrospective cohort study from 20 ICUs spanning 3 years found a similar overall survival of children with or without malignancies (95 vs $96 \%$; $p=0.2$ ). However, a large number $(72 \%)$ of cancer patients were admitted after elective surgery and survived to discharge. Among these children, if mechanical ventilation and vasopressors were needed, then the survival decreased to $71 \% .^{5}$ In children who were not admitted postoperatively and who needed both mechanical ventilation and vasopressors, the survival rate dropped to $46 \%$. A small single-center retrospective study from Pakistan found that though the overall mortality of children with cancer was only $32.4 \%$, and this was largely due to low mortality (16.7\%) in children admitted for postoperative care. ${ }^{14}$ In an old study from Israel, the overall mortality was $44 \%$, where the study cohort mainly consisted of children with solid tumors (52\%), intracranial tumors (30\%), and extracranial (22\%). ${ }^{15}$
Eighty-six (43\%) patients presented to us with acute respiratory failure (ARF), and their overall survival rate was $52 \%$. The need for IMV was an independent predictor of hospital mortality [odds ratio (OR), 14.637; 95\% confidence interval (Cl): 1.298-165.053; $p<0.030]$. This is consistent with several other reports. ${ }^{1,3,11,15}$ Wösten-van Asperen et al. conducted a systematic review that included 16,853 admissions of children with cancer from 31 studies. They found that the risk of death was significantly higher (OR, 18.49; $\mathrm{Cl}, 13.79-24.78$; $p<0.001$ ) when mechanical ventilation was required. ${ }^{6}$

Thirty-five children in our study were initially given NIV, of which 19 (54\%) patients failed NIV and required IMV. Out of these, 11 children subsequently died, suggesting that delay in intubation and IMV is likely to increase mortality. In a small study in adult immunocompromised patients, the early use of NIV in hypoxic respiratory failure was shown to reduce the need for intubation and mechanical ventilation and also to reduce serious complications and mortality. ${ }^{16}$ Later, Pancera et al. described similar encouraging results of NIV as the first line of therapy in pediatric patients with malignancies. NIV was used successfully in $74 \%$ of children. ${ }^{17}$ They cautioned against its use in patients who had high severity of illness and cardiovascular dysfunction. Pistra et al. reported that the success of NIV is highest in the presence of single organ failure, and the number of organ failures at ICU admission is a strong predictor of failure of NIV (OR, 5.26; 95\% Cl: $1.7-16.4 ; p=0.004) .{ }^{18}$ Garcio-Salida reported the outcomes of 69 pediatric oncology patients with 88 episodes of ARF admitted to PICU, over 4 years. ${ }^{19}$ They reported that high-flow oxygen nasal cannula (HFNC), NIV, or $\mathrm{O}_{2}$ by nasal cannula in that order were the types of respiratory support offered at admission. IMV was required in 47 patients, of whom 37 patients needed IMV because primary support that was offered failed. A large proportion of children, who were given HFNC or NIV, did not need mechanical ventilation. However, if these two therapies failed, the outcomes worsened. They suggested the need for further studies for assessing the utility of noninvasive respiratory support in pediatric oncology patients.

In our study, of the 82 patients who later developed circulatory failure and hypotension, 55 patients died (64.7\%).On univariate analysis, use of vasopressors ( 19 vs $54 ; p<0.001$ ) and inotropes ( 2 vs $13 ; p<0.001$ ) was associated with increased ICU mortality (Table 2), but not on multivariate analysis .On multivariate analysis, the need for inotropic (dobutamine) support was associated with increased mortality. Fifteen children needed inotropic support; of these, 5 patients had solid tumors, while 10 had hematological malignancies. All patients had cardiac dysfunction with reduced myocardial contractility on admission. Two patients developed septic shock during ICU stay. Their mean ICU LOS was 2.8 
( \pm 2.81 ) days. Only two patients survived to be discharged from ICU, of which one died in hospital subsequently. On multivariate analysis, the need for inotropes, that is, dobutamine (OR, 9.806; $95 \%$ $\mathrm{Cl}:-1.222$ to $78.660 ; p=0.032$ ), was an independent predictor of mortality (Table 2). Most of the previous studies have reported the use of vasoactive medications and mortality, but none of them have given discrete effects of either vasopressors or inotropes on survival of patients. Butt et al looked at the outcomes of 133 patients with hematological malignancies admitted to PICU. Of the 36 patients who had a peripheral circulatory failure, the causes were sepsis (28), drug-induced cardiomyopathy (7), and anemia (1). Twenty-seven (75\%) of these patients died. In a study in 1996, van Veen reported that in pediatric oncology patients, when patients on mechanical ventilation needed inotropic support, the mortality increased to $69 \%$, as compared to $55 \%$ with ventilation alone. Dalton et al. reported that of the 226 nonoperative patients in their cohort, 58 $(42.6 \%)$ patients received vasoactive medications. They further reported that if the patients needed both mechanical ventilation and vasoactive medications, the survival came down to $46 \%$ compared to mechanical ventilation (93\%) and vasoactive agents (89\%), either used alone. ${ }^{5}$ Tamburro et al. reported that when the reason for mechanical ventilation was a cardiovascular failure, the odds ratio (5.00,95\% Cl: 2.60-10.06) for mortality was higher in the pediatric oncology than transplant recipients $(4.70,95 \% \mathrm{Cl}: 2.14$, 10.92). ${ }^{20}$ A small study in 36 pediatric oncology patients looked at risk factors predictive of mortality and found that need for inotropic support along with sepsis, organ system dysfunction, and the need for mechanical ventilation predicted mortality. Of the 23 patients who received inotropic support, $74 \%$ died. ${ }^{21}$ In the study discussed above about NIV support in children, hemodynamic failure was a risk factor for mortality if associated with respiratory failure. ${ }^{19}$

We found that coagulopathy was an independent predictor of hospital mortality (OR, 3.86; $95 \% \mathrm{Cl}:-1.568$ to $9.51 ; p<0.003$ ).
Apart from direct effect, the immune-mediated mechanisms of chemotherapeutic agents also cause bone marrow hypoplasia. In addition, apoptotic effects on megakaryocytes, splenic sequestration due to hepatotoxicity of the drugs, inhibition of platelet release and platelet-derived growth factor, and toxic mediators released in the bone marrow all affect thrombocyte counts. ${ }^{22}$ In critically ill patients, the commonest etiology for thrombocytopenia is sepsis, caused by multiple mechanisms, such as immune-mediation, sequestration of platelets, interaction between platelet receptors, and as a part of disseminated intravascular coagulation (DIC). ${ }^{23}$ Raised INR is common in critically ill and is caused by many conditions, such as liver disease, DIC, vitamin $\mathrm{K}$ deficiency, inherited deficiency coagulation factors due to production defects, etc. ${ }^{24}$ Coagulopathy and its causes in pediatric patients have been extensively reviewed by Parker. ${ }^{25}$ To our knowledge, this is the first study to find a correlation between the presence of coagulopathy and mortality in pediatric oncology patients. The incidence of thrombocytopenia and raised INR was more common in patients with hematological malignancies, and they needed FFP and platelet transfusions (Table 4).

Various studies show that the number of multi-organ failures is directly related to prognosis and mortality exceeds $70 \%$, if three or more organ systems are involved. ${ }^{10,15,21}$ We found that children with septic shock requiring mechanical ventilation and vasopressors had significantly higher mortality. Nearly $12 \%$ of our patients had cardiac arrest within 24 hours of ICU admission, which probably indicates high severity of illness. In our cohort, we found that as compared to children with solid tumors, patients with hematological malignancies had a higher number of organ failures and greater mortality, though we did not use any severity of illness score, since none has been validated in the oncology cohort (Table 5).

The strengths of our study are that it is the largest data set from a single center from India. The limitations of our study are

Table 4: Hematological disturbances, intervention, and mortality $(n=200)$

\begin{tabular}{|c|c|c|c|c|c|}
\hline $\begin{array}{l}\text { ICU admission } \\
\text { diagnosis/intervention }\end{array}$ & Total, $n$ (\%) & $\begin{array}{c}\text { Hematological } \\
\text { malignancies, } n \text { (\%) }\end{array}$ & Mortality, n (\%) & $\begin{array}{c}\text { Solid organ } \\
\text { malignancies, } n \text { (\%) }\end{array}$ & Mortality, $n$ (\%) \\
\hline Coagulopathy & $78(39)$ & $56(71.79)$ & $35(44.87)$ & $22(28.21)$ & $10(45.45)$ \\
\hline FFP transfusions & $23(11.5)$ & $16(69.56)$ & $11(47.82)$ & $7(30.44)$ & $3(42.85)$ \\
\hline Platelet transfusions & $59(29.5)$ & $46(77.96)$ & $28(47.45)$ & $13(22.04)$ & $7(53.84)$ \\
\hline
\end{tabular}

Table 5: Variables predicting mortality (univariate analysis)

\begin{tabular}{lrcc}
\hline Variables & Odds ratio & \multicolumn{1}{c}{$\mathrm{Cl}$} & $p$ value \\
\hline Hypotension & 6.50 & $3.59-11.74$ & $<0.001$ \\
Cardiac arrest within 24 hours of ICU admission & 15.47 & $1.94-123.06$ & 0.002 \\
Need for vasopressors & 8.43 & $4.51-15.76$ & $<0.001$ \\
Need for inotropes & 10.35 & $2.27-47.06$ & 0.001 \\
NIV & 0.58 & $0.29-1.18$ & 0.315 \\
IMV & 12.17 & $4.20-35.28$ & $<0.001$ \\
Renal failure & 4.41 & $1.52-12.71$ & 0.007 \\
Neutropenia & 1.61 & $0.91-2.87$ & 0.44 \\
Coagulopathy & 2.73 & $1.55-4.79$ & 0.001 \\
Tumor lysis syndrome & 1.20 & $0.39-3.71$ & 0.783 \\
Altered sensorium/seizures/encephalopathy & 0.83 & $0.45-1.54$ & 0.568 \\
Enterocolitis & 2.25 & $1.07-4.74$ & 0.012 \\
Significant pulmonary bleed & 12.09 & $1.48-98.41$ & 0.019 \\
\hline
\end{tabular}


the exclusion of hematopoietic stem cell transplant patients and the lack of formal calculation of a severity score, owing to the retrospective nature of the study.

We hope that this data will help in guiding the pediatric intensivists in risk stratification of children with cancer by identifying patients who are likely to have poor outcomes and also identifying patients who may benefit from ICU admission. This will help in better allocation of resources and explaining the prognosis of the child to the family members. Further research is needed in validating the scoring systems in children with malignancy, since no such score is currently validated in the Indian population.

\section{Conclusion}

In this retrospective cohort of 200 children with malignancies, we found that children with hematologic cancer had significantly higher hospital mortality as compared to those with solid tumors. The need for mechanical ventilation, use of inotrope infusion, and coagulopathy were independent predictors of mortality.

\section{ORCID}

Shilpushp J Bhosale 자 https://orcid.org/0000-0002-0290-0526

Malini Joshi ㄴ https://orcid.org/0000-0001-5051-9474

Vijaya P Patil @ https://orcid.org/0000-0002-5177-5696

Amol T Kothekar (1) https://orcid.org/0000-0002-7751-1314

Sheila Nainan Myatra @ https://orcid.org/0000-0001-6761-163X

Jigeeshu V Divatia (1) https://orcid.org/0000-0001-7384-4886

Atul P Kulkarni @ https://orcid.org/0000-0002-5172-7619

\section{References}

1. Van Veen A, Karstens A, van der Hoek AC, Tibboel D, Hählen K, van der Voort $E$, et al. The prognosis of oncologic patients in the pediatric intensive care unit. Intensive Care Med 1996;22(3):41. DOI: 10.1007/ BF01712243.

2. Hallahan AR, Shaw PJ, Rowell G, O'Connell A, Schell D, Gillis J. Improved outcomes of children with malignancy admitted to a pediatric intensive care unit. Crit Care Med 2000;28(11):3718-3722. DOI: 10.1097/00003246-200011000-00030.

3. Rr P, Tan EEK, Sultana R, Thoon KC, Chan MY, Lee JH, et al. Critical illness epidemiology and mortality risk in pediatric oncology. Pediatr Blood Cancer 2020;67(6):e28242. DOI: 10.1002/pbc.28242.

4. Tilford JM, Roberson PK, Lensing S, Fiser DH. Differences in pediatric ICU mortality risk over time. Crit Care Med 1998;26(10):1737-1743. DOI: 10.1097/00003246-199810000-00032.

5. Dalton HJ, Slonim AD, Pollack MM. MultiCenter outcome of pediatric oncology patients requiring intensive care. Pediatr Hematol Oncol 2003;20(8):643-649. DOI: 10.1080/08880010390243095.

6. Wösten-van Asperen RM, van GestelJPJ, van Grotel M, Tschiedel E, Dohna-Schwake C, Valla FV, et al. POKER (PICU Oncology Kids in Europe Research group) research consortium. PICU mortality of children with cancer admitted to pediatric intensive care unit a systematic review and meta-analysis. Crit Rev Oncol Hematol 2019;142:153-163. DOI: 10.1016/j.critrevonc.2019.07.014.

7. Butt W. What is the outcome of children admitted to intensive care? This is the most important question we need to answer! Pediatr Crit Care Med 2017;18(3):292-293. DOI: 10.1097/PCC.0000000000001080.

8. Butt W, Barker G, Walker C, Gillis J, Kilham H, Stevens M. Outcome of children with hematologic malignancy who are admitted to an intensive care unit. Crit Care Med 1988;16(8):761-764. DOI: 10.1097/00003246-198808000-00005.

9. Verlaat $\mathrm{CW}$, Wubben $\mathrm{N}$, Visser IH, Hazelzet JA, van der Hoeven J, Lemson J, et al. SKIC (Dutch collaborative PICU research network).
Retrospective cohort study on factors associated with mortality in high-risk pediatric critical care patients in the Netherlands. BMC Pediatr 2019;19(1):274. DOI: 10.1186/s12887-019-1646-9.

10. Ali AM, Sayed HA, Mohammed MM. The outcome of critically ill pediatric cancer patients admitted to the pediatric intensive care unit in a tertiary university oncology center in a developing country: a 5-year experience. J Pediatr Hematol Oncol 2016;38(5):355-359. DOI: 10.1097/MPH.0000000000000523.

11. Zinter MS, DuBois SG, Spicer A, Matthay K, Sapru A. Pediatric cancer type predicts infection rate, need for critical care intervention, and mortality in the pediatric intensive care unit. Intensive Care Med 2014;40(10):1536-1544. DOI: 10.1007/s00134-014-3389-2.

12. López-Herce J, Sancho L, Martinón JM. Study of paediatric intensive care units in Spain. Spanish Society of Paediatric Intensive Care. Intensive Care Med 2000;26(1):62-68. DOI: 10.1007/s001340050013.

13. Garrido MM, GarridoRQ, Cunha TN, Ehrlich S, Martins IS. Comparison of epidemiological, clinical and microbiological characteristics of bloodstream infection in children with solid tumours and haematological malignancies. Epidemiol Infect 2019;147:e298. DOI: 10.1017/S0950268819001845.

14. Akhtar N, Fadoo Z, Panju S, Haque A. Outcome and prognostic factors seen in pediatric oncology patients admitted in PICU of a developing country. Indian J Pediatr 2011;78(8):969-972. DOI: 10.1007/s12098011-0391-3.

15. Ben Abraham R, Toren A, Ono N, Weinbroum AA, Vardi A, Barzilay Z, et al. Predictors of outcome in the pediatric intensive care units of children with malignancies. J Pediatr Hematol Oncol 2002;24(1): 23-26. DOI: 10.1097/00043426-200201000-00007.

16. Hilbert G, Gruson D, Vargas F, Valentino R, Gbikpi-Benissan G, Dupon M, et al. Noninvasive ventilation in immunosuppressed patients with pulmonary infiltrates, fever, and acute respiratory failure. $\mathrm{N}$ Engl J Med 2001;344(7):481-487. DOI: 10.1056/NEJM200102153440703.

17. Pancera $C F$, Hayashi $M$, Fregnani JH, NegriEM, Deheinzelin $D$, de Camargo B. Noninvasive ventilation in immunocompromised pediatric patients: eight years of experience in a pediatric oncology intensive care unit. J Pediatr Hematol Oncol 2008;30(7):533-538. DOI: 10.1097/MPH.0b013e3181754198.

18. Piastra M, De Luca D, Marzano L, Stival E, Genovese O, Pietrini D, Conti $G$. The number of failing organs predicts non-invasive ventilation failure in children with ALI/ARDS. Intensive Care Med 2011;37(9):1510-1516. DOI: 10.1007/s00134-011-2308-z.

19. García-Salido A, Mastro-Martínez I, Cabeza-Martín B, Oñoro G, NietoMoro M, Iglesias-Bouzas MI, et al. Respiratory failure in children with hemato-oncological diseases admitted to the PICU: a single-center experience. J Pediatr Hematol Oncol 2015;37(6):449-454. DOI: 10.1097/MPH.0000000000000377.

20. Tamburro RF, Barfield RC, Shaffer ML, Rajasekaran S, Woodard P, Morrison RR, et al. Changes in outcomes (1996-2004) for pediatric oncology and hematopoietic stem cell transplant patients requiring invasive mechanical ventilation. Pediatr Crit Care Med 2008;9(3): 270-277. DOI: 10.1097/PCC.0b013e31816c7260.

21. Dursun O, Hazar V, Karasu GT, Uygun V, Tosun O, Yesilipek A. Prognostic factors in pediatric cancer patients admitted to the pediatric intensive care unit. J Pediatr Hematol Oncol 2009;31(7):481-484. DOI: 10.1097/ MPH.0b013e3181a330ef.

22. Tamamyan G, Danielyan S, Lambert MP. Chemotherapy induced thrombocytopenia in pediatric oncology. Crit Rev Oncol Hematol 2016;99:299-307. DOI: 10.1016/j.critrevonc.2016.01.005.

23. Ghimire S, Ravi S, Budhathoki R, Arjyal L, Hamal S, Bista A, et al. Current understanding and future implications of sepsis induced thrombocytopenia. Eur J Haematol 2021;106(3):301-305. DOI: 10.1111/ ejh.13549.

24. Yang R, Moosavi L. Prothrombin time [updated July 10, 2020]. In: StatPearls [Internet]. Treasure Island (FL): StatPearls Publishing; 2020. Available from: https://www.ncbi.nlm.nih.gov/books/NBK544269/.

25. Parker RI. Coagulopathies in the PICU: DIC and liver disease. Crit Care Clin 2013;29(2):319-333. DOI: 10.1016/j.ccc.2012.12.003. 


\section{Appendix I}

\section{Definitions}

\section{Septic Shock}

The American College of Critical Care Medicine defines sepsis as the presence of hypothermia or hyperthermia plus clinical signs of inadequate tissue perfusion including any of the following: Decreased or altered mental status; capillary refill time $>2$ seconds, diminished pulses, and mottled cool extremities (cold shock); flash capillary refill, bounding peripheral pulses, wide pulse pressure (warm shock); and urine output $<1 \mathrm{~mL} / \mathrm{kg} /$ hour. Hypotension is not necessary for the clinical diagnosis of septic shock, but its presence in a child with clinical suspicion of infection is confirmatory.

Davis AL, Carcillo JA, Aneja RK, Deymann AJ, Lin JC, Nguyen TC, et al., American College of Critical Care Medicine clinical practice parameters for hemodynamic support of pediatric and neonatal septic shock. Crit Care Med 2017;45(6):1061-1093. DOI: 10.1097/ CCM.0000000000002425.

\section{Hypotension}

As per Pediatric Sepsis Consensus Conference 2005 (PSCC) Criteria.

\section{Cardiovascular Dysfunction}

Despite administration of isotonic intravenous fluid bolus $\geq 40 \mathrm{~mL} /$ $\mathrm{kg}$ in 1 hour

- Decrease in BP (hypotension) $<5$ th percentile for age or systolic $\mathrm{BP}<2$ SD below normal for age

OR

- Need for vasoactive drug to maintain BP in normal range (dopamine $\geq 5 \mu \mathrm{g} / \mathrm{kg} /$ minute or dobutamine, epinephrine, or norepinephrine at any dose

OR

- Two of the following

Unexplained metabolic acidosis: Base deficit $\geq 5.0 \mathrm{mEq} / \mathrm{L}$ Increased arterial lactate $>2$ times upper limit of normal Oliguria: Urine output $\leq 0.5 \mathrm{~mL} / \mathrm{kg} / \mathrm{hr}$
Prolonged capillary refill:_ 5 seconds

Core to peripheral temperature gap $33^{\circ} \mathrm{C}$

Goldstein B, Giroir B, Randolph A; International Consensus Conference on Pediatric Sepsis. International pediatric sepsis consensus conference: definitions for sepsis and organ dysfunction in pediatrics. Pediatr Crit Care Med 2005;6(1):2-8. DOI: 10.1097/01. PCC.0000149131.72248.E6.

\section{Renal Failure}

KDIGO criteria.

Sutherland SM, Byrnes JJ, Kothari M, Longhurst CA, Dutta S, Garcia P, et al., AKI in hospitalized children: Comparing the pRIFLE, AKIN, and KDIGO definitions. Clin J Am Soc Nephrol 2015;10(4):554561. DOI: $10.2215 / C J N .01900214$.

\section{Neutropenia}

Absolute neutrophil count $<1000 / \mathrm{mm}^{3}$.

\section{Coagulopathy}

Presence of low platelets $\left(<50 \times 10^{3} / \mathrm{mm}^{3}\right)$ and/or raised INR $(>1.4)$

\section{Cardiac Failure}

Screening echocardiography showing moderate cardiac function $(<35 \%)$ with need for inotropes.

\section{Respiratory Failure}

Acute respiratory failure describes any impairment in oxygenation or ventilation in which the arterial oxygen tension falls below $60 \mathrm{~mm} \mathrm{Hg}$ (acute hypoxemia), the carbon dioxide tension rises above $50 \mathrm{~mm} \mathrm{Hg}$ (acute hypercarbia, hypercapnia), and the $\mathrm{pH}$ drops below 7.35, or both. For patients who have underlying chronic respiratory failure, acute hypercarbia can be diagnosed by an increase in $\mathrm{PCO}_{2}$ by $20 \mathrm{~mm} \mathrm{Hg}$ from baseline. From a functional standpoint, respiratory failure is defined as the inability of the respiratory system to meet the metabolic needs of the tissues.

Nitu ME, Eigen H. Respiratory failure. Pediatr Rev 2009;30(12): 470-477; quiz 478. DOI: 10.1542/pir.30-12-470. 